\title{
Arrest of rapid crack propagation in polymer pipes
}

\author{
P. FLÜELER, M. FARSHAD \\ EMPA Department Plastics Composites
}

\section{INTRODUCTION}

Under certain thermal and service conditions, internally pressurized pipes may experience the phenomenon of rapid crack propagation (RCP) [1]. The critical internal pressure which causes RCP in the pipe depends on the ambient temperature, pipe dimensions, material properties, pipe processing, residual stresses, ageing, service conditions, properties of pressurizing fluid, supporting and/or embedding conditions, repair, and maintenance conditions $[2,3])$. The present design philosophy is based mainly on the strength criteria for the internal pressure [4]; accordingly, for higher internal pressure, a larger pipe wall thickness is chosen. On the other hand, the research already cited has shown that pipes with thicker walls may be more prone to RCP than pipes with thinner walls. The motivation of the present work has been to develop mechanisms which would inhibit a running crack and thus strengthen the pipes, in particular in existing pipelines, against dynamic fracture and to enhance their degree of safety.

\section{CRACK-STOPPERS}

A crack-stopper has, in principle, the function of absorbing the energy which would otherwise be spent in opening the crack and in its propagation along the pipeline. On the other hand, the energy release rate, i.e., the energy available for crack growth, depends on the material and the geometrical properties of the pipe. Thus, the search to develop a crack-stopping mechanism may be pursued in one of the following directions: (1) development of tougher material, (2) choice of proper interplay among the geometrical parameters, (3) combination of the concepts stated in (1) and (2), and/or (4) regulation of the service conditions; e.g., lowering of internal pressure.

Development of 'crack-stoppers' at EMPA has been part of an on-going investigation of the dynamic behaviour of polymeric pipes [5-8]. To this end, a number of research and development projects have been carried out. The design of the EMPA crack arrester utilizes the knowledge that, below a certain wall thickness, the so-called critical thickness, RCP cannot occur; hence, as crack arresters, the designs envisaged included multi-layer pipe segments. These crack arresting systems absorb dynamic energy through additional flexibility and energy storage. Each of these systems can be installed along the PE pipe at some distances apart. Varieties of crack-stoppers which were constructed on this design principle included: a multi-layer pipe segment with partial adhesion between the layers, a pipe segment with

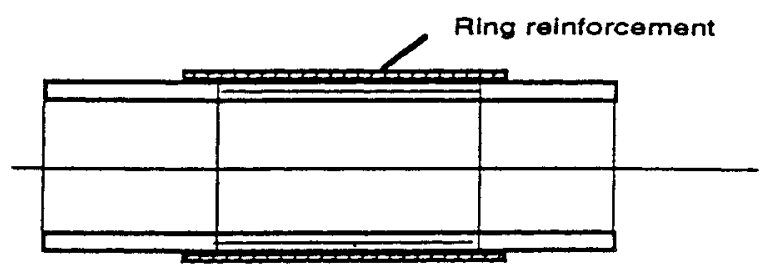

(a) A multi- layer plpe segment

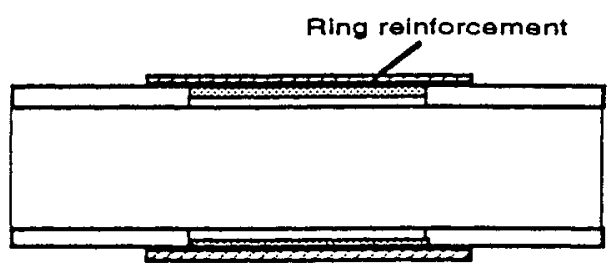

(b) Reduced thickness with damping element and ring relnforcement

Fig. 1 A number of variants for crack arrest along a PE-HD pipe.

reduced wall thickness, and a multi-layer pipe segment with damping element and reinforcing rings. Fig. 1 shows some of these variants; other variants are modifications of these schemes.

\section{PIPE SAMPLES AND TESTING PROCEDURE}

The gas pipe samples with and without crack-stoppers tested were of PE-HD material. The nominal external diameter of the pipes was $225 \mathrm{~mm}$ and their nominal wall thickness was about $20.5 \mathrm{~mm}$; the standard diameter ratio (SDR) of these pipes was 11. The length of each pipe sample was $1500 \mathrm{~mm}$; the length of crack-stoppers was of the same order of magnitude as the pipe diameter. For thermal conditioning of the samples, a climatecontrolled test room was used. It was possible to adjust the temperature of this chamber from outside; in this system, a minimum temperature of $-40^{\circ} \mathrm{C}$ could be achieved.

The crack-stopping systems were tested at $0^{\circ} \mathrm{C}$. Previously, pipes of the same grade, but with no crack-stoppers had been tested at the same temperature; based on such tests, the critical internal pressure at $0^{\circ} \mathrm{C}$ was found to be about 1.75 bar [8]. To test the effectiveness of the crack arresting mechanisms, an internal pressure considerably higher than the critical pressure of regular pipes was chosen. Accordingly, the pipe samples equipped with crack arresters (installed at the mid-length of the sample) were subjected to $2.5,5.0$, and 7.0 bar internal pressures. 


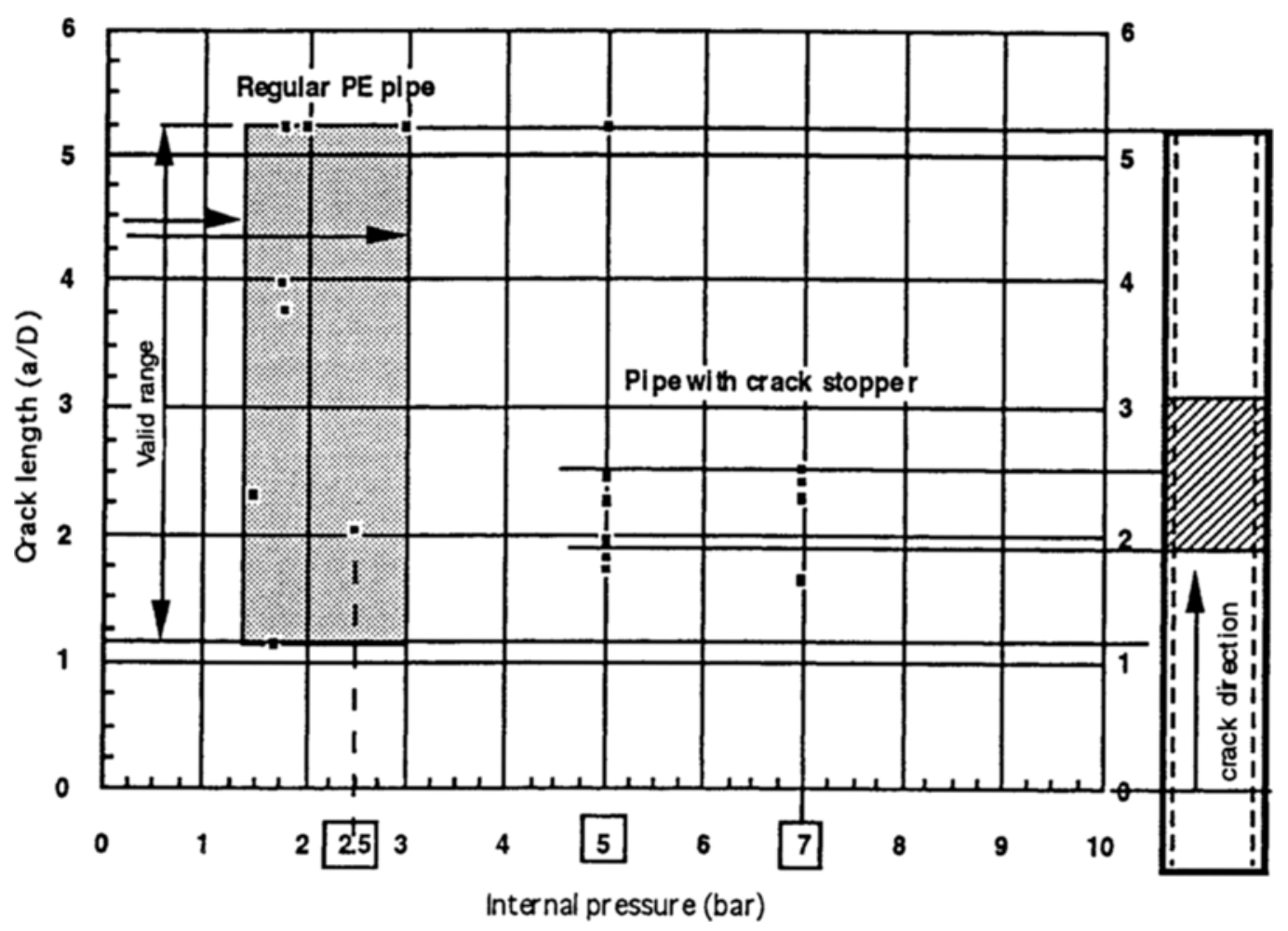

Fig. 2 Variation of crack length with internal pressure for a PE-HD pipe with and without crack-stoppers.

\section{EXPERIMENTAL RESULTS}

The experiments with the crack-stopper systems which followed the principle of wall thickness reduction and/or lamination proved to be successful. All the samples tested

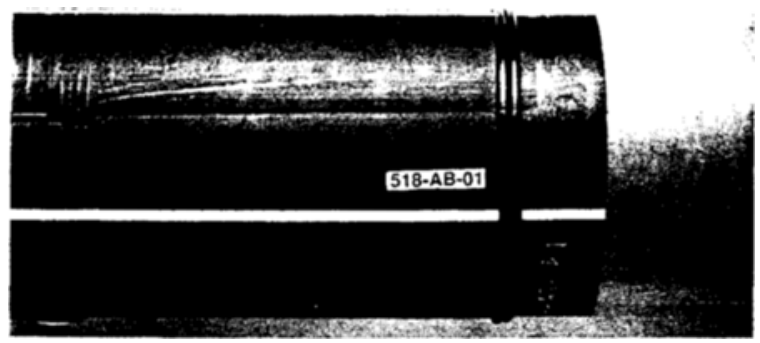

(a)

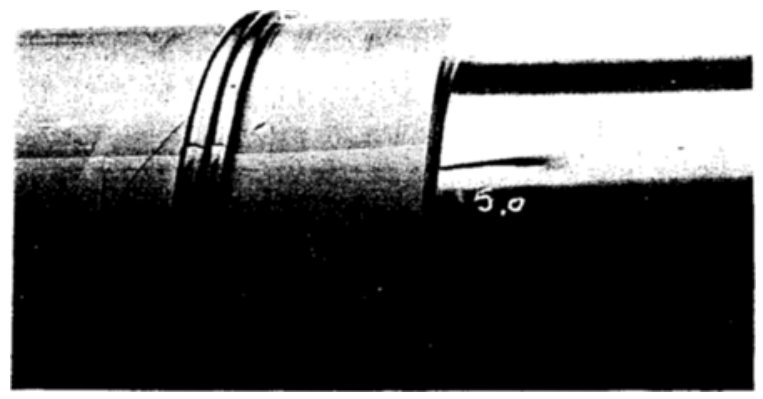

(b)

Fig. 3 Arrested crack in PE pipes from S4 Tests at $0^{\circ} \mathrm{C}$ : (a) crack path deflected ahead of the stopper zone at 7 bar internal pressure (EMPA Photo 101186), and (b) arrest by plastification of the crack tip in the stopper zone at 5 bar (EMPA Photo 101016). withstood the energy of 5 bar and 7 bar internal pressure. Under these conditions, a brittle crack would run through the whole length of a plain tube sample. In the samples with crack-stoppers, the running crack, upon its arrival at the crack-stopper, virtually stopped and did not propagate further. Moreover, it was observed that the cracking mode at the crack tip changed from a brittle to a ductile mode. Fig. 2 shows changes in crack length with internal pressure; it depicts the effectiveness of crack-stoppers in the pipe. Fig. 3 presents photographs of two of the crack-stoppers after the test, indicating the function of the crack-stoppers in arresting the crack ahead of and in the stopper zone.

\section{DISCUSSION AND PRACTICAL REALIZATION}

The crack-stopper variants developed and tested here while having proved very effective in arresting the running cracks have the advantage of simplicity in construction and ease of installation along new or existing pipelines. To arrive at practical recommendations, further tests must be carried out on the samples with similar strengthening. Moreover, the parameters related to the geometry of the crack-stopper should be varied so that an optimum construction may be achieved. Further investigations should include testing of these systems under controlled field conditions. Morepver, the crack-stopping mechanisms must also fulfil the same requirements as imposed on the pipeline; those include burst resistance and long-term strength against internal 
pressure at different temperature levels. An attempt has been made to develop crack-stopping mechanisms that are easy to install in existing pipelines; nevertheless, to achieve an optimum installation, these mechanisms may require further improvements.

\section{REFERENCES}

1. 'ISO TC 138: CD 13'477, Resistance to crack propagation: Determination of the critical pressure for rapid crack propagation (Small Scale Steady State [S4])' (ISO).

2. Yayla, P., 'Rapid crack propagation in polyethylene gas pipes', Ph.D. Thesis, Department of Mechanical Engineering, Imperial College, London, January, 1991.

3. Leevers, P. S., Venizelos, G. and Ivankovic, A., 'Rapid crack propagation along pressurised $\mathrm{PE}$ pipe: small-scale testing and numerical modelling', 'Proc. Plastics Pipes VIII', (The Plastics and Rubber Institute, The Netherlands, 1992) pp. D1/7-1-11.
4. 'ISO/TR 9080: Methods of extrapolation of hydrostatic rupture data to determinate long-term hydrostatic strength of thermoplastic pipe materials' (ISO).

5. Farshad, M. and Flüeler, P., 'Crack propagation in cylindrical shells and pipes', Int. J. Engng Fracture Mech. 44 (1993) 937-947.

6. Flüeler, P. and M. Farshad, M., 'Schnelle Rissausbreitungeine Besonderheit im Bruchverhalten von KunststoffRohren', gwa (Gas, Wasser, Abwasser, Schweizerischen Vereins des Gas-und Wasserfaches), 72 Jahrgang (1992) pp. $350-352$.

7. Farshad, M. and Flüeler, P., 'Crack propagation in prestressed plates', Int. J. Engng Fracture Mech. (1995) in press.

8. Idem, 'Dynamic fracture toughness of thermoplastic pipes rapid crack propagation in PE-HD pipes', EMPA internal Report No. 150'317/1, September 1994.

\section{Fibre optic sensors for civil structures monitoring}

\section{U. SENNHAUSER, R. BRÖNNIMANN, PH. M. NELLEN}

EMPA Department Electronics/Metrology

\section{INTRODUCTION}

In recent years an increasing quantity of advanced composites and other new materials as well as construction methods have been applied in buildings, prestressed tendons, bridges, etc. Without long term experience of their ageing behaviour it is important for us to apply efficient tools for monitoring their quality and long term stability.

Sensors used today broadly for this purpose are, e.g., electrical resistance strain gauges, displacement transducers, extensometers and optical systems for special applications. In academic research much attention has been given to the very attractive idea of using optical fibres for sensing all kinds of physical, chemical and biological effects. Techniques using back or forward scattered light, polarization states or intensities in multi or mono mode fibres have been investigated, although few are competitive with conventional methods.

Of particular interest in civil engineering are fibre optic sensors for measuring strain and displacements and for detecting cracks $[1,2]$. Before they will become commercially acceptable, they have to be made more efficient, reliable, accurate, cheaper and easier to apply than the well established methods mentioned above.

In this work, low coherence interferometry is used for absolute strain measurements using embedded fibre Fabry-Perot sensors and fibre gratings with broad bandwidths. It matches the range and resolution of electrical resistance strain gauges, and its multiplexing capabilities make it a technically and economically promising tool for distributed sensing.

\section{FIBRE OPTIC SENSING SYSTEM}

Low coherence or white light interferometry attracts increasing attention for measuring absolute distances with interferometric resolutions of a few nanometres $(\mathrm{nm})$. In long term field monitoring it is mandatory to use such an absolute method to avoid interrupt free measurements as required in normal laser interferometry and in measuring local strain distributions, e.g., $1 \mu \mathrm{m} \mathrm{m}^{-1}$ with a local resolution of $1 \mathrm{~cm}$, requires the measurement of relative displacements of $10 \mathrm{~nm}$.

A schematic of an experimental set-up is shown in Fig. 1. A broadband superluminescent light diode (SLD) has a sufficiently short coherence length of $26 \mu \mathrm{m}$ for unambiguous identification of the zero fringe and discrimination between different length sensing elements $\left(d_{F P} \ldots 3\right)$ in the fibre in a parallel or serial multiplexing scheme. Low coherence demodulation is performed with a scanning Michelson interferometer with a moveable mirror $\mathbf{M}_{2}$ whose position is controlled permanently by

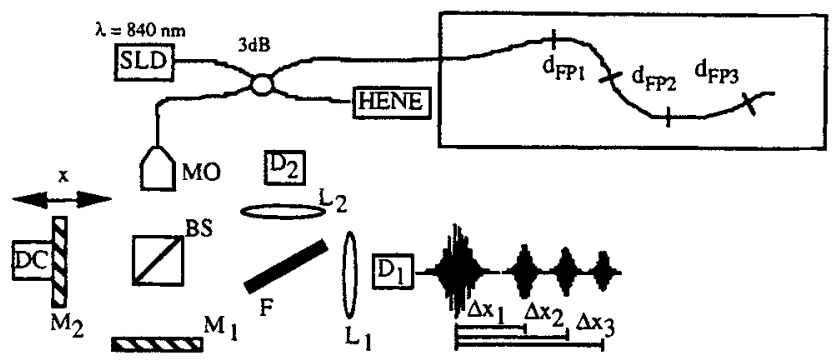

Fig. 1 Schematic drawing of the scanning Michelson interferometer with serially embedded FP sensor elements. MO, microscope objective; BS, beamsplitter; DC, DC motor; $M_{1,2}$, mirrors; $D_{1,2}$, detectors; $L_{1,2}$, lenses; $F$, filter. 